\title{
Global status of tetracycline resistance among clinical isolates of Vibrio cholerae: a systematic review and meta-analysis
}

\author{
Mohammad Hossein Ahmadi ${ }^{*}$ (D)
}

\begin{abstract}
Background: There has been an increasing resistance rate to tetracyclines, the first line treatment for cholera disease caused by $V$. cholera strains, worldwide. The aim of the present study was to determine the global status of resistance to this class of antibiotic among $V$. cholera isolates.

Methods: For the study, electronic databases were searched using the appropriate keywords including:'Vibrio,',cholera,' 'Vibrio cholerae, 'V. cholerae,', resistance,',antibiotic resistance,',antibiotic susceptibility', 'antimicrobial resistance', 'antimicrobial susceptibility', 'tetracycline', and 'doxycycline'. Finally, after some exclusion, 52 studies from different countries were selected and included in the study and meta-analysis was performed on the collected data.

Results: The average resistance rate for serogroup $\mathrm{O} 1$ to tetracycline and doxycycline was $50 \%$ and $28 \%$, respectively $(95 \% \mathrm{Cl})$. A high level of heterogeneity $\left(I^{2}>50 \%, p\right.$-value $\left.<0.05\right)$ was observed in the studies representing resistance to tetracycline and doxycycline in $\mathrm{O} 1$ and non-O1, non-O139 serogroups. The Begg's tests did not indicate the publication bias ( $p$-value $>0.05$ ). However, the Egger's tests showed some evidence of publication bias in the studies conducted on serogroup 01 .

Conclusions: The results of the present study show that the overall resistance to tetracyclines is relatively high and prevalent among $V$. cholerae isolates, throughout the world. This highlights the necessity of performing standard antimicrobial susceptibility testing prior to treatment choice along with monitoring and management of antibiotic resistance patterns of $V$. cholerae strains in order to reduce the emergence and propagation of antibiotic resistant strains as well as the failure of treatment.
\end{abstract}

Keywords: Vibrio cholerae, Tetracycline, Doxycycline, Resistance

\section{Introduction}

Cholera is an ancient infectious disease mainly affecting developing countries. The disease is capable to spread across many countries leading to vast pandemics and becoming a major public health concern throughout the world [1].
*Correspondence: mhahmadi@shahed.ac.ir Department of Microbiology, Faculty of Medicine, Shahed University, Tehran, Iran
The causative agent of this life threatening diarrheal disease is Vibrio cholerae secreting the cholera toxin. Two major cholera toxin-producing serogroups of this bacterial pathogen, $\mathrm{O} 1$ and $\mathrm{O} 139$, have potential to spread and cause epidemic as well as pandemic disease [2]. The serogroup $\mathrm{O} 1$ has two biotypes, classical and El Tor, and each biotype has three serotypes including Ogawa, Inaba, and Hikojima [1,3].

The main stay of management of cholera (acute gastroenteritis) is urgent fluid replacement; however, the use of an appropriate antibiotic is necessary to eliminate the original author(s) and the source, provide a link to the Creative Commons licence, and indicate if changes were made. The images or other third party material in this article are included in the article's Creative Commons licence, unless indicated otherwise in a credit line to the material. If material is not included in the article's Creative Commons licence and your intended use is not permitted by statutory regulation or exceeds the permitted use, you will need to obtain permission directly from the copyright holder. To view a copy of this licence, visit http://creativecommons.org/licenses/by/4.0/. The Creative Commons Public Domain Dedication waiver (http://creativecommons.org/publicdomain/zero/1.0/) applies to the data made available in this article, unless otherwise stated in a credit line to the data. 
bacteria, lessen the duration of illness, and control the disease [4].

Tetracyclines (tetracycline and doxycycline) have long been the antibiotics of choice for treating severe cholera effectively worldwide, except for young children and pregnant women [5]. However, tetracycline resistant strains of $V$. cholerae are being increasingly reported worldwide. These resistant strains have been responsible for major epidemics in some countries and geographical areas such as Latin America, Tanzania, Bangladesh, and Zaire [6].

To date, numerous studies have reported the different antibiotic resistance patterns for $V$. cholerae isolates throughout the world. Nevertheless, the overall status of the resistance to teracyclines among the strains is not intensively studied.

The present study was conducted to determine the global status of resistance to the antibiotics of tetracycline family, including tetracycline and doxycycline, among different $V$. cholerae isolates using a systematic review and meta-analysis according to the Preferred Reporting Items for Systematic Reviews and Meta-Analyses (PRISMA) statement [7].

\section{Methods}

\section{Search strategies}

The electronic databases, including OVID databases, PubMed, Web of Science, Scopus, MEDLINE, EMBASE, Cochrane Library, as well as Google Scholar, were searched for papers reporting the resistance rate for different Vibrio cholerae isolates to the antibiotics of tetracyclines family from December 1980 to April 2020. The search was restricted to original research articles throughout the world, published in English using the following keywords with the help of Boolean operators (AND, OR): 'Vibrio', 'cholera', 'Vibrio cholerae, ' $V$. cholerae,' 'resistance,' 'antibiotic resistance', 'antibiotic susceptibility', 'antimicrobial resistance', 'antimicrobial susceptibility', 'tetracycline', and 'doxycycline.' References from reviewed articles were also searched for more information.

\section{Inclusion and exclusion criteria}

Included studies were all original research articles as well as some letter to editors presenting the resistance rates for Vibrio cholerae isolates to the tetracyclines including tetracycline and doxycycline.

Excluded articles were those that: (1) had no sufficient data to be analyzed; (2) reported antibiotic resistance of Vibrio species other than V. cholerae; (3) studied resistance to antibiotics other than tetracyclines; and (4) tested environmental isolates of the bacterium instead of clinical ones; for example, the strains isolated from wastewater, water supplies, river, aquaculture water, fishery products, as well as seafood.

Review articles, congress abstracts, studies reported in languages other than English, meta-analyses or systematic reviews, duplicate publications of the same study and articles available only in abstract form were also excluded.

\section{Data extraction}

The data extracted from each study included first author's name, year of publication, geographical area of study (country), clinical sample (specimen type), serogroup, biotype, and serotype of the isolates, number of investigated isolates (sample size), method of susceptibility testing, and number of isolates resistant to each antibiotic.

\section{Statistical analysis}

The data were analyzed using Comprehensive MetaAnalysis Software Version 2.0 (Biostat, Englewood, NJ, USA). The resistance rate was reported by $95 \%$ confidence intervals (CIs).

Cochrane Q-statistic test and $I^{2}$ test were performed to estimate heterogeneity between studies, and in all calculations of which $I^{2}$ was above $50 \%$, the random effect model was chosen to estimate the average rate because of its conservative summary estimate; otherwise, the fixed effect model was applied. To assess possible publication bias, a funnel plot, Begg's rank correlation and Egger's weighted regression methods were used. Two-tailed $p<0.05$ was considered indicative of a significant publication bias. The relative weight for each study was also calculated.

\section{Results}

A total of 138 articles were collected for assessment. Through the first screening, 15 articles were excluded on the basis of the title evaluation, as nine of them were duplicate publications of the same study, and six have titles irrelevant to the present study. By the second assessment, nine papers were discarded because they had represented the study of Vibrio species other than $V$. cholerae, or were review articles. Finally, after fulltext evaluation, 62 studies were ruled out because they had reported resistance to antibiotics other than tetracyclines, used environmental isolates of the bacterium instead of clinical ones, and/or had no sufficient data. Therefore, 52 articles published between 1980-2020 were selected and included in the final analysis (Fig. 1 and Table 1).

The included studies were carried out in 20 different countries, majority of which (38 studies) located in Asia, 12 in Europe, one in Caribbean, and one in Oceania (Table 1). 
English databases: OVID databases, PubMed, Scopus, Web of Science, MED LINE, EMBASE, Cochrane Library, and Google $S$ cholar.

$$
N=138
$$

Excluded:

Duplicates: $n=9$

Title not relevant: $n=6$

Abstract evaluation:

$$
n=123
$$

Excluded:

other Vibrio spp. : $\mathrm{n}=5$

Review articles: $n=4$

Full-text evaluation:

$n=114$

Excluded:

Antibiotics other than

tetra cyclines" $: n=22$

Environmental (non-clinical)

isolates $^{* * *}: \mathbf{n =} 31$

No sufficient data: $\mathbf{n}=\mathbf{9}$

\section{Artides included in meta-analysis:}

$$
\mathrm{N}=\mathbf{5 2}
$$

Fig. 1 Flow chart of the literature search, systematic review and study selection. *Articles representing study of Vibrio species other than V. cholerae; ${ }^{* *}$ Articles reported resistance to antibiotics other than tetracyclines; ${ }^{* *}$ Studies using environmental instead of clinical isolates of $V$. cholerae 
Table 1 Studies included in meta-analysis after final evaluation

\begin{tabular}{|c|c|c|c|c|c|c|c|c|c|}
\hline \multirow[t]{2}{*}{ References } & \multirow[t]{2}{*}{ Pub. year } & \multirow[t]{2}{*}{ Country } & \multirow[t]{2}{*}{ Source } & \multirow[t]{2}{*}{$\begin{array}{l}\text { Serogroup/ } \\
\text { biotype }\end{array}$} & \multirow[t]{2}{*}{ Serotype } & \multirow[t]{2}{*}{$\begin{array}{l}\text { No. of } \\
\text { isolates }\end{array}$} & \multicolumn{2}{|c|}{$\begin{array}{l}\text { No. of resistant } \\
\text { isolate (\%) }\end{array}$} & \multirow{2}{*}{$\begin{array}{l}\text { Method of } \\
\text { susceptibility } \\
\text { testing }\end{array}$} \\
\hline & & & & & & & TET & DOX & \\
\hline $\begin{array}{l}\text { Olipher et al. } \\
\text { [8] }\end{array}$ & 2020 & Kenya & Stool & Non-O1 & ND & 98 & $64(65.3)$ & NM & $\begin{array}{l}\text { Kirby-Bauer disk } \\
\text { diffusion }\end{array}$ \\
\hline Kale et al. [9] & 2020 & India & Stool & O1/El Tor & Ogawa & 109 & $0(0)$ & $0(0)$ & $\begin{array}{l}\text { Kirby-Bauer disk } \\
\text { diffusion }\end{array}$ \\
\hline $\begin{array}{l}\text { Abana et al. } \\
{[10]}\end{array}$ & 2019 & Ghana & ND & O1/El Tor & Ogawa & 40 & $14(35)$ & $6(15)$ & $\begin{array}{l}\text { Kirby-Bauer disk } \\
\text { diffusion }\end{array}$ \\
\hline $\begin{array}{l}\text { Zereen et al. } \\
\text { [11] }\end{array}$ & 2019 & Bangladesh & Stool & ND/ND & ND & 3 & $2(66.7)$ & NM & $\begin{array}{l}\text { Kirby-Bauer disk } \\
\text { diffusion }\end{array}$ \\
\hline $\begin{array}{c}\text { Sreedhara and } \\
\text { Mohan [12] }\end{array}$ & 2019 & India & Stool & O1/El Tor & Ogawa & $\begin{array}{l}\text { 74 TET, } 41 \\
\text { DOX }\end{array}$ & $19(25.7)$ & $10(24.4)$ & $\begin{array}{l}\text { Kirby-Bauer disk } \\
\text { diffusion }\end{array}$ \\
\hline Dua et al. [13] & 2018 & India & Stool & $\begin{array}{l}\text { Non-O1, non- } \\
\text { O139 }\end{array}$ & ND & 71 & $29(40.9)$ & $63(88.7)$ & $\begin{array}{l}\text { Kirby-Bauer disk } \\
\text { diffusion }\end{array}$ \\
\hline $\begin{array}{l}\text { Uddin et al. } \\
\text { [14] }\end{array}$ & 2018 & Bangladesh & Stool & O1/ND & $\begin{array}{l}43 \text { Ogawa \& } \\
15 \text { Inaba }\end{array}$ & 58 & $17(29.3)$ & $10(17.2)$ & $\begin{array}{l}\text { Kirby-Bauer disc } \\
\text { diffusion }\end{array}$ \\
\hline $\begin{array}{l}\text { Fernández- } \\
\text { Abreu et al. } \\
\text { [15] }\end{array}$ & 2017 & Cuba & Stool & $\begin{array}{l}\text { Non-O1, non- } \\
\text { O139 }\end{array}$ & ND & 125 & $5(4)$ & $1(0.8)$ & $\begin{array}{l}\text { Kirby-Bauer disk } \\
\text { diffusion }\end{array}$ \\
\hline Shah et al. [16] & 2017 & Pakistan & $\begin{array}{l}\text { Stool \& vomi- } \\
\text { tus }\end{array}$ & ND/ND & ND & 131 & $13(9.9)$ & NM & $\begin{array}{l}\text { Kirby-Bauer disk } \\
\text { diffusion }\end{array}$ \\
\hline $\begin{array}{l}\text { Dengo-Baloi } \\
\text { et al. [17] }\end{array}$ & 2017 & Mozambique & Rectal swabs & O1/El Tor & Ogawa & 159 & $79(50)$ & $89(56)$ & $\begin{array}{l}\text { Kirby-Bauer disk } \\
\text { diffusion }\end{array}$ \\
\hline Patil et al. [18] & 2017 & India & Stool & O1/El Tor & Ogawa & 106 & $10(9.4)$ & NM & $\begin{array}{l}\text { Kirby-Bauer disk } \\
\text { diffusion }\end{array}$ \\
\hline Jain et al. [19] & 2016 & India & Rectal swabs & O1/El Tor & Ogawa & 27 & $27(100)$ & NM & $\begin{array}{l}\text { Kirby-Bauer disk } \\
\text { diffusion \& } \\
\text { broth dilution }\end{array}$ \\
\hline Hajia et al. [20] & 2016 & Iran & ND & O1/ND & $\begin{array}{l}\text { Ogawa \& } \\
\text { Inaba }\end{array}$ & 192 & 115 (59.9) & $\begin{array}{l}\text { NM } \\
\text { NM }\end{array}$ & $\begin{array}{l}\text { Liofilchem Test } \\
\text { Strip }\end{array}$ \\
\hline $\begin{array}{l}\text { Torane et al. } \\
\text { [1] }\end{array}$ & 2016 & India & Stool & O1/El Tor & $\begin{array}{c}407 \text { Ogawa \& } \\
32 \text { Inaba }\end{array}$ & 439 & $55(12.5)$ & NM & $\begin{array}{l}\text { Kirby-Bauer disk } \\
\text { diffusion }\end{array}$ \\
\hline $\begin{array}{l}\text { Gupta et al. } \\
\text { [21] }\end{array}$ & 2016 & Nepal & Stool & O1/El Tor & Ogawa & 31 & $0(0)$ & $0(0)$ & Agar dilution \\
\hline $\begin{array}{l}\text { Masoumi-Asl } \\
\text { et al. [22] }\end{array}$ & 2016 & Iran & Stool & O1/ND & Inaba & 60 & $60(100)$ & NM & $\begin{array}{l}\text { Liofilchem Test } \\
\text { Strip }\end{array}$ \\
\hline Irfan et al. [23] & 2016 & Pakistan & Stool & $\begin{array}{l}\text { Non-O1, non- } \\
\text { O139 }\end{array}$ & ND & 233 & $5(2.1)$ & NM & $\begin{array}{l}\text { Kirby-Bauer disk } \\
\text { diffusion }\end{array}$ \\
\hline Afzali et al. [24] & 2016 & Iran & Stool & $\begin{array}{l}\text { Non-O1, non- } \\
\text { O139 }\end{array}$ & ND & 96 & $3(3.1)$ & $3(3.1)$ & $\begin{array}{l}\text { Kirby-Bauer disk } \\
\text { diffusion }\end{array}$ \\
\hline Kar et al. [6] & 2015 & India & Rectal swabs & O1/El Tor & Ogawa & 35 & $35(100)$ & NM & $\begin{array}{l}\text { Kirby-Bauer disk } \\
\text { diffusion }\end{array}$ \\
\hline Ukaji et al. [25] & 2015 & Nigeria & Stool & O1/ND & ND & 63 & $53(84.1)$ & NM & $\begin{array}{l}\text { Kirby-Bauer disk } \\
\text { diffusion }\end{array}$ \\
\hline $\begin{array}{l}\text { Tabatabaei } \\
\text { and Khora- } \\
\text { shad [26] }\end{array}$ & 2015 & Iran & Stool & O1/ND & Inaba & 48 & $29(60.4)$ & NM & $\begin{array}{l}\text { Kirby-Bauer disk } \\
\text { diffusion }\end{array}$ \\
\hline $\begin{array}{l}\text { Barati et al. } \\
\text { [27] }\end{array}$ & 2015 & Iran & Rectal swabs & O1/El Tor & Ogawa & 239 & $36(15.1)$ & $10(4.2)$ & $\begin{array}{l}\text { Kirby-Bauer disk } \\
\text { diffusion }\end{array}$ \\
\hline $\begin{array}{l}\text { Mishra et al. } \\
\text { [28] }\end{array}$ & 2015 & India & Stool & O1/El Tor & Ogawa & 44 & $0(0)$ & NM & $\begin{array}{l}\text { Kirby-Bauer disk } \\
\text { diffusion }\end{array}$ \\
\hline $\begin{array}{l}\text { Kuma et al. } \\
\text { [29] }\end{array}$ & 2014 & Ghana & $\begin{array}{l}\text { Stool \& vomi- } \\
\text { tus }\end{array}$ & O1/ND & ND & 275 & $43(15.6)$ & $40(14.5)$ & $\begin{array}{l}\text { Kirby-Bauer disk } \\
\text { diffusion }\end{array}$ \\
\hline $\begin{array}{l}\text { Mercy et al. } \\
\text { [30] }\end{array}$ & 2014 & Kenya & ND & O1/El Tor & $\begin{array}{l}\text { Inaba (most } \\
\text { commen) \& } \\
\text { Ogawa }\end{array}$ & 44 & $0(0)$ & $0(0)$ & $\begin{array}{l}\text { Kirby-Bauer disk } \\
\text { diffusion }\end{array}$ \\
\hline
\end{tabular}


Table 1 (continued)

\begin{tabular}{|c|c|c|c|c|c|c|c|c|c|}
\hline \multirow[t]{2}{*}{ References } & \multirow[t]{2}{*}{ Pub. year } & \multirow[t]{2}{*}{ Country } & \multirow[t]{2}{*}{ Source } & \multirow[t]{2}{*}{$\begin{array}{l}\text { Serogroup/ } \\
\text { biotype }\end{array}$} & \multirow[t]{2}{*}{ Serotype } & \multirow[t]{2}{*}{$\begin{array}{l}\text { No. of } \\
\text { isolates }\end{array}$} & \multicolumn{2}{|c|}{$\begin{array}{l}\text { No. of resistant } \\
\text { isolate (\%) }\end{array}$} & \multirow{2}{*}{$\begin{array}{l}\text { Method of } \\
\text { susceptibility } \\
\text { testing }\end{array}$} \\
\hline & & & & & & & TET & DOX & \\
\hline $\begin{array}{l}\text { Mahmud et al. } \\
\text { [31] }\end{array}$ & 2014 & Sierra Leone & Rectal swabs & O1/El Tor & Ogawa & 15 & $0(0)$ & $0(0)$ & $\begin{array}{l}\text { Kirby-Bauer disk } \\
\text { diffusion \& } \\
\text { E-test }\end{array}$ \\
\hline $\begin{array}{l}\text { Murhekar et al. } \\
\text { [32] }\end{array}$ & 2013 & $\begin{array}{l}\text { Papua New } \\
\text { Guinea }\end{array}$ & $\begin{array}{l}\text { Stool \& rectal } \\
\text { swabs }\end{array}$ & O1/El Tor & Ogawa & 299 & $29(9.7)$ & NM & $\begin{array}{l}\text { Kirby-Bauer disk } \\
\text { diffusion }\end{array}$ \\
\hline Tran et al. [33] & 2012 & Vietnam & ND & O1/El Tor & Ogawa & 100 & $29(29)$ & $0(0)$ & E-test strips \\
\hline Sang et al. [34] & 2012 & Kenya & Stool & O1/ND & ND & 4 & $0(0)$ & NM & $\begin{array}{l}\text { Kirby-Bauer disk } \\
\text { diffusion }\end{array}$ \\
\hline $\begin{array}{l}\text { Mandal et al. } \\
\text { [35] }\end{array}$ & 2012 & India & Stool & O1/El Tor & $\begin{array}{l}150 \text { Ogawa \& } \\
4 \text { Inaba }\end{array}$ & 154 & $26(16.9)$ & NM & $\begin{array}{l}\text { Agar dilution \& } \\
\text { E-test }\end{array}$ \\
\hline $\begin{array}{l}\text { Shujatullah } \\
\text { et al. [36] }\end{array}$ & 2012 & India & Stool & O1/ND & $\begin{array}{l}\text { Ogawa (most } \\
\text { commen) \& } \\
\text { Inaba }\end{array}$ & 66 & $9(13.6)$ & $8(12.1)$ & $\begin{array}{l}\text { Kirby-Bauer disk } \\
\text { diffusion }\end{array}$ \\
\hline $\begin{array}{l}\text { Borkakoty } \\
\text { et al. [37] }\end{array}$ & 2012 & India & Rectal swabs & O1/El Tor & $\begin{array}{c}24 \text { Ogawa \& } \\
16 \text { Inaba }\end{array}$ & 40 & $16(40)$ & NM & $\begin{array}{l}\text { Kirby-Bauer disk } \\
\text { diffusion }\end{array}$ \\
\hline Das et al. [38] & 2011 & India & $\begin{array}{l}\text { Stool \& rectal } \\
\text { swabs }\end{array}$ & O1/ND & $\begin{array}{l}\text { Ogawa (most } \\
\text { common), } \\
\text { Inaba, Hiko- } \\
\text { jima }\end{array}$ & 238 & $41(17.2)$ & NM & $\begin{array}{l}\text { Kirby-Bauer disk } \\
\text { diffusion \& } \\
\text { broth dilution }\end{array}$ \\
\hline Karki et al. [39] & 2011 & Nepal & Stool & O1/El Tor & Ogawa & 57 & $0(0)$ & NM & $\begin{array}{l}\text { Kirby-Bauer disk } \\
\text { diffusion }\end{array}$ \\
\hline $\begin{array}{l}\text { Rahbar et al. } \\
\text { [40] }\end{array}$ & 2010 & Iran & $\begin{array}{l}\text { Stool \& rectal } \\
\text { swabs }\end{array}$ & O1/El Tor & $\begin{array}{l}199 \text { Inaba \& } 21 \\
\text { Ogawa }\end{array}$ & 220 & $0(0)$ & $0(0)$ & $\begin{array}{l}\text { Kirby-Bauer disk } \\
\text { diffusion }\end{array}$ \\
\hline $\begin{array}{l}\text { Abera et al. } \\
\text { [41] }\end{array}$ & 2010 & Ethiopia & Stool & O1/ND & Inaba & 81 & $5(6.2)$ & $0(0)$ & $\begin{array}{l}\text { Kirby-Bauer disk } \\
\text { diffusion }\end{array}$ \\
\hline $\begin{array}{l}\text { Supawat et al. } \\
\text { [42] }\end{array}$ & 2009 & Thailand & $\begin{array}{l}\text { Stool \& } \\
\text { rectal swabs, } \\
\text { blood }\end{array}$ & $\begin{array}{l}\text { O1/ND } \\
\text { O139 } \\
\text { Non-O1, non- } \\
\text { O139 }\end{array}$ & $\begin{array}{l}1032 \text { Inaba \& } \\
43 \text { Ogawa } \\
\text { ND } \\
\text { ND }\end{array}$ & $\begin{array}{l}1075 \\
41 \\
22\end{array}$ & $\begin{array}{l}16(1.5) \\
\mathrm{NM} \\
3(13.6)\end{array}$ & $\begin{array}{l}\text { NM } \\
\text { NM } \\
\text { NM }\end{array}$ & $\begin{array}{l}\text { Kirby-Bauer disk } \\
\text { diffusion }\end{array}$ \\
\hline $\begin{array}{l}\text { Keramat et al. } \\
\text { [43] }\end{array}$ & 2008 & Iran & Stool & O1/El Tor & Inaba & 60 & $14(23.3)$ & $20(33.3)$ & $\begin{array}{l}\text { Kirby-Bauer disk } \\
\text { diffusion }\end{array}$ \\
\hline $\begin{array}{l}\text { Roychowd- } \\
\text { hury et al. } \\
\text { [44] }\end{array}$ & 2008 & India & $\begin{array}{l}\text { Stool \& rectal } \\
\text { swabs }\end{array}$ & O1/ND & $\begin{array}{l}\text { Inaba \& } \\
\text { Ogawa }\end{array}$ & 51 & $9(17.6)$ & $\begin{array}{l}\text { NM } \\
\text { NM }\end{array}$ & $\begin{array}{l}\text { Kirby-Bauer disk } \\
\text { diffusion }\end{array}$ \\
\hline $\begin{array}{l}\text { Mandomando } \\
\text { et al. [45] }\end{array}$ & 2007 & Mozambique & Rectal swabs & O1/ND & Ogawa & 75 & $73(97.3)$ & NM & $\begin{array}{l}\text { Kirby-Bauer disk } \\
\text { diffusion }\end{array}$ \\
\hline $\begin{array}{l}\text { Faruque et al. } \\
\text { [5] }\end{array}$ & 2007 & Bangladesh & ND & O1/ND & $\begin{array}{c}762 \text { Ogawa \& } \\
535 \text { Inaba }\end{array}$ & 1297 & $711(54.8)$ & NM & $\begin{array}{l}\text { Kirby-Bauer disk } \\
\text { diffusion }\end{array}$ \\
\hline Rafi et al. [46] & 2004 & Pakistan & Stool & $\begin{array}{l}\text { O1/ } 66 \text { El Tor \& } \\
57 \text { Classical }\end{array}$ & ND & 123 & $37(30.1)$ & NM & $\begin{array}{l}\text { Kirby-Bauer disk } \\
\text { diffusion }\end{array}$ \\
\hline $\begin{array}{l}\text { Tjaniadi et al. } \\
\text { [47] }\end{array}$ & 2003 & Indonesia & $\begin{array}{l}\text { Stool \& rectal } \\
\text { swabs }\end{array}$ & $\begin{array}{l}\text { O1/ND } \\
\text { Non-O1, non- } \\
\text { O139 }\end{array}$ & $\begin{array}{l}N D \\
N D\end{array}$ & $\begin{array}{l}1044 \\
68\end{array}$ & $\begin{array}{l}12(1.2) \\
6(8.8)\end{array}$ & $\begin{array}{l}\text { NM } \\
\text { NM }\end{array}$ & $\begin{array}{l}\text { Kirby-Bauer disk } \\
\text { diffusion }\end{array}$ \\
\hline $\begin{array}{l}\text { Dromigny } \\
\text { et al. [48] }\end{array}$ & 2002 & Madagascar & Stool & O1/El Tor & ND & 351 & $55(15.7)$ & NM & $\begin{array}{l}\text { Kirby-Bauer disk } \\
\text { diffusion }\end{array}$ \\
\hline $\begin{array}{l}\text { Sabeena et al. } \\
\text { [49] }\end{array}$ & 2001 & India & Stool & O1/El Tor & Ogawa & 25 & $2(8.0)$ & NM & $\begin{array}{l}\text { Kirby-Bauer disk } \\
\text { diffusion }\end{array}$ \\
\hline $\begin{array}{l}\text { Iwanaga et al. } \\
\text { [50] }\end{array}$ & 2000 & Laos & ND & O1/El Tor & Ogawa & 99 & $95(95.9)$ & NM & Agar dilution \\
\hline $\begin{array}{l}\text { Urassa et al. } \\
\text { [51] }\end{array}$ & 2000 & Tanzania & Stool & O1/ND & ND & 181 & $42(23.2)$ & NM & $\begin{array}{l}\text { Kirby-Bauer disk } \\
\text { diffusion }\end{array}$ \\
\hline Garg et al. [52] & 2000 & India & ND & $\begin{array}{l}\text { O1/ND } \\
\text { O139 } \\
\text { Non-O1, non- } \\
\text { O139 }\end{array}$ & $\begin{array}{l}\text { Ogawa } \\
\text { ND } \\
\text { ND }\end{array}$ & $\begin{array}{l}326 \\
314 \\
200\end{array}$ & $\begin{array}{l}8(2.5) \\
8(2.5) \\
55(27.5)\end{array}$ & $\begin{array}{l}\text { NM } \\
\text { NM } \\
\text { NM }\end{array}$ & $\begin{array}{l}\text { Kirby-Bauer disk } \\
\text { diffusion }\end{array}$ \\
\hline
\end{tabular}


Table 1 (continued)

\begin{tabular}{|c|c|c|c|c|c|c|c|c|c|}
\hline \multirow[t]{2}{*}{ References } & \multirow[t]{2}{*}{ Pub. year } & \multirow[t]{2}{*}{ Country } & \multirow[t]{2}{*}{ Source } & \multirow[t]{2}{*}{$\begin{array}{l}\text { Serogroup/ } \\
\text { biotype }\end{array}$} & \multirow[t]{2}{*}{ Serotype } & \multirow[t]{2}{*}{$\begin{array}{l}\text { No. of } \\
\text { isolates }\end{array}$} & \multicolumn{2}{|c|}{$\begin{array}{l}\text { No. of resistant } \\
\text { isolate (\%) }\end{array}$} & \multirow{2}{*}{$\begin{array}{l}\text { Method of } \\
\text { susceptibility } \\
\text { testing }\end{array}$} \\
\hline & & & & & & & TET & DOX & \\
\hline $\begin{array}{l}\text { Ranjit et al. } \\
\text { [53] }\end{array}$ & 2000 & Malaysia & ND & ND/ND & ND & 24 & $8(33.3)$ & NM & $\begin{array}{l}\text { Kirby-Bauer disk } \\
\text { diffusion }\end{array}$ \\
\hline Dhar et al. [54] & 1996 & Bangladesh & Stool & $\begin{array}{l}\text { O1/El Tor } \\
\text { O139 }\end{array}$ & $\begin{array}{l}\text { ND } \\
\text { ND }\end{array}$ & $\begin{array}{l}110 \\
132\end{array}$ & $\begin{array}{l}46(42) \\
0(0)\end{array}$ & $\begin{array}{l}1(0.9) \\
0(0)\end{array}$ & $\begin{array}{l}\text { Kirby-Bauer disk } \\
\text { diffusion }\end{array}$ \\
\hline $\begin{array}{l}\mathrm{Ng} \text { and Taha } \\
\text { [55] }\end{array}$ & 1994 & Malaysia & Rectal swabs & O1/EITor & Ogawa & 3 & $3(100)$ & NM & $\begin{array}{l}\text { Kirby-Bauer disk } \\
\text { diffusion }\end{array}$ \\
\hline Glass et al. [56] & 1980 & Bangladesh & Stool & O1/ND & $\begin{array}{l}\text { Inaba \& } \\
\text { Ogawa }\end{array}$ & 256 & $54(21.1)$ & NM & $\begin{array}{l}\text { Kirby-Bauer disk } \\
\text { diffusion \& } \\
\text { broth dilution }\end{array}$ \\
\hline
\end{tabular}

NM not measured; ND not determined; TET tetracycline; DOX doxycycline

Of 52 articles included, 40 had studied only O1 serogroup all of which reported El Tor biotype, five had detected only non-O1, non-O139 serogroup, four had investigated O1, O139, and/or non-O1, non-O139 serogroups simultaneously, and three did not determined the serogroups of isolated $V$. cholerae. From the included studies, only one had detected and tested classical biotype beside the El Tor one.

The most commonly collected samples for assessment in the included studies were stool, and rectal swabs, but other samples included vomitus and blood (for isolation of non O1, non O139 V. cholerae).

The included studies tested antimicrobial susceptibility to tetracycline and doxycycline for the serogroups O1 (44 and 16 studies, respectively), O139 (two and one studies, respectively), and non-O1, non-O139 (eight and three studies, respectively) of $V$. cholerae. From the studies conducted on serogroup O1, 19 detected only Ogawa, three only Inaba, and 12 detected both serotypes, simultaneously. Only one study detected Hikojima serotype along with other two serotypes, concurrently. The remaining studies conducted on serogroup O1 did not determine the serotypes of their isolates.
The studies mainly used Kirby-Bauer disk diffusion method for susceptibility testing, but other techniques were broth and agar dilution, E-test, and Liofilchem Test Strip.

The number of $V$. cholerae isolates investigated (sample sizes) in the studies varied from 3-1297. The range of antibiotic resistance as well as the pooled resistance rate for $V$. cholerae isolates (serogroups $\mathrm{O} 1, \mathrm{O} 139$, and non-O1, non-O139) to tetracycline and doxycycline are shown in Table 2.

The average resistance rate for serogroup $\mathrm{O} 1$ to tetracycline and doxycycline was $50 \%$ and $28 \%$, respectively (95\% CI). Figures $2 \mathrm{a}-\mathrm{c}$ and $3 \mathrm{a}-\mathrm{c}$ show the forest plots of the meta-analysis for resistance rate of different serogroups of $V$. cholerae to the antibiotics. A high level of heterogeneity $\left(I^{2}>50 \%, p\right.$-value $\left.<0.05\right)$ was observed in the studies representing resistance to tetracycline and doxycycline in $\mathrm{O} 1$ and non-O1, non-O139 serogroups; however, the number of included studies conducted on the antimicrobial resistance of $\mathrm{O} 139$, as well as nonO1, non-O139 serogroups to tetracycline and doxycycline was fewer than 10 and insufficient for an accurate analysis.

Table 2 Meta-analysis results for resistance rate of each $V$. cholera serogroup in included studies

\begin{tabular}{|c|c|c|c|c|c|c|c|c|c|c|}
\hline \multirow[t]{2}{*}{ Serogroup } & \multirow[t]{2}{*}{$\begin{array}{l}\text { Number of } \\
\text { studies }\end{array}$} & \multirow[t]{2}{*}{ Antibiotic } & \multicolumn{3}{|c|}{$\begin{array}{l}\text { Resistance rate }(\%) \\
(95 \% \mathrm{Cl})\end{array}$} & \multicolumn{2}{|c|}{ Heterogeneity test } & \multicolumn{2}{|c|}{$\begin{array}{l}\text { Begg's test }{ }^{* *} \\
p \text {-value (two- } \\
\text { tailed) }\end{array}$} & \multirow[t]{2}{*}{$\begin{array}{l}\text { Egger's test*** } \\
p \text {-value (two- } \\
\text { tailed) }\end{array}$} \\
\hline & & & Min & Max & Pooled* (range) & $I^{2}(\%)$ & $p$-value & $\mathbf{a}$ & b & \\
\hline O1 & $\begin{array}{l}44 \\
16\end{array}$ & $\begin{array}{l}\text { TET } \\
\text { DOX }\end{array}$ & $\begin{array}{l}0 \\
0\end{array}$ & $\begin{array}{l}100 \\
56\end{array}$ & $\begin{array}{l}0.2(0.1-0.3) \\
0.07(0.03-0.1)\end{array}$ & $\begin{array}{l}96.9 \\
92.5\end{array}$ & $\begin{array}{l}<0.001 \\
<0.001\end{array}$ & $\begin{array}{l}0.2 \\
0.7\end{array}$ & $\begin{array}{l}0.2 \\
0.8\end{array}$ & $\begin{array}{l}0.03 \\
0.005\end{array}$ \\
\hline 0139 & $\begin{array}{l}2 \\
1\end{array}$ & $\begin{array}{l}\text { TET } \\
\text { DOX }\end{array}$ & $\begin{array}{l}0 \\
N A\end{array}$ & $\begin{array}{l}2.5 \\
\text { NA }\end{array}$ & $\begin{array}{l}0.02(0.01-0.04) \\
0.004(0.0-0.06)\end{array}$ & $\begin{array}{l}43 \\
0.0\end{array}$ & $\begin{array}{l}0.2 \\
1.0\end{array}$ & $\begin{array}{l}\text { NA } \\
\text { NA }\end{array}$ & $\begin{array}{l}\text { NA } \\
\text { NA }\end{array}$ & $\begin{array}{l}\text { NA } \\
\text { NA }\end{array}$ \\
\hline Non-O1, non-O139 & $\begin{array}{l}8 \\
3\end{array}$ & $\begin{array}{l}\text { TET } \\
\text { DOX }\end{array}$ & $\begin{array}{l}2.1 \\
0.8\end{array}$ & $\begin{array}{l}65.3 \\
88.7\end{array}$ & $\begin{array}{l}0.1(0.05-0.3) \\
0.1(0.001-0.9)\end{array}$ & $\begin{array}{l}95.5 \\
97.7\end{array}$ & $\begin{array}{l}<0.001 \\
<0.001\end{array}$ & $\begin{array}{l}0.2 \\
0.6\end{array}$ & $\begin{array}{l}0.3 \\
1.0\end{array}$ & $\begin{array}{l}0.06 \\
0.3\end{array}$ \\
\hline
\end{tabular}

TET tetracycline; DOX doxycycline; a: Kendall's tau without continuity correction; b: Kendall's tau with continuity correction; *Pooled resistance rate; **Begg and Mazumdar rank correlation; ${ }^{* * *}$ Egger's regression intercept; NA: not applicable 


\begin{tabular}{|c|c|c|c|c|c|c|c|}
\hline \multirow[t]{2}{*}{ Study name } & \multicolumn{7}{|c|}{ Statistics for each study } \\
\hline & Total & $\begin{array}{c}\text { Event } \\
\text { rate }\end{array}$ & $\begin{array}{l}\text { Lower } \\
\text { limit }\end{array}$ & $\begin{array}{c}\text { Upper } \\
\text { limit }\end{array}$ & $z$-value & $p$-value & $\begin{array}{c}\text { Relative } \\
\text { weight }\end{array}$ \\
\hline Kale et al. & $0 / 109$ & 0.005 & 0.000 & 0.068 & -3.802 & 0.000 & 1.27 \\
\hline Abana et al. & $14 / 40$ & 0.350 & 0.219 & 0.508 & -1.867 & 0.062 & 2.64 \\
\hline Sreedhara \& Mohan & $19 / 74$ & 0.257 & 0.170 & 0.368 & -3.994 & 0.000 & 2.70 \\
\hline Uddin et al. & $17 / 58$ & 0.293 & 0.191 & 0.422 & -3.052 & 0.002 & 2.68 \\
\hline Dengo-Baloi et al. & $79 / 159$ & 0.497 & 0.420 & 0.574 & -0.079 & 0.937 & 2.77 \\
\hline Patil et al. & $10 / 106$ & 0.094 & 0.052 & 0.167 & -6.807 & 0.000 & 2.63 \\
\hline Jain et al. & $27 / 27$ & 0.982 & 0.770 & 0.999 & 2.808 & 0.005 & 1.26 \\
\hline Hajia et al. & $115 / 192$ & 0.599 & 0.528 & 0.666 & 2.724 & 0.006 & 2.77 \\
\hline Torane et al. & $55 / 439$ & & & & -13.479 & 0.000 & 2.78 \\
\hline Gupta e & & 0.016 & 0.001 & 0.206 & -2.907 & 0.004 & 1.26 \\
\hline sl et al. & 601 & 0.992 & 0.882 & 0.999 & & 0.001 & 1.26 \\
\hline Kar et al. & $35 / 35$ & 0.986 & 0.813 & 0.999 & 2.993 & 0.003 & 1.26 \\
\hline Ukaji et al. & $53 / 63$ & 0.841 & 0.729 & 0.912 & 4.837 & 0.000 & 2.62 \\
\hline Tabatabaei \& Khoras! & ad $29 / 48$ & 0.604 & 0.461 & 0.731 & 1.433 & 0.152 & 2.67 \\
\hline Barati et al. & $36 / 239$ & 0.151 & 0.111 & 0.202 & -9.565 & 0.000 & 2.76 \\
\hline Mishra et al. & $0 / 44$ & 0.011 & 0.001 & 0.154 & -3.156 & 0.002 & 1.26 \\
\hline Kuma et al. & $43 / 275$ & 0.156 & 0.118 & 0.204 & -10.152 & 0.000 & 2.76 \\
\hline Mercy et al. & $0 / 44$ & 0.011 & 0.001 & 0.154 & -3.156 & 0.002 & 1.26 \\
\hline Mahmud et al. & $0 / 15$ & 0.031 & 0.002 & 0.350 & -2.390 & 0.017 & 1.25 \\
\hline Murhe & 291 & 0.097 & 0.068 & 0.136 & -11. & 0.000 & 2.75 \\
\hline Tran e & $29 / 1$ & 0.290 & 0.210 & 0.386 & -4.063 & 0.000 & 2.73 \\
\hline Sang et al. & $0 / 4$ & 0.100 & 0.006 & 0.674 & -1.474 & 0.140 & 1.20 \\
\hline Mandal et al. & $26 / 154$ & 0.169 & 0.118 & 0.236 & -7410 & 0.000 & 2.73 \\
\hline Shuja & & & & & & & \\
\hline Borkakoty & $16 / 40$ & 0.400 & 0.262 & 0.557 & -1.256 & & 2.64 \\
\hline Das et al. & $41 / 238$ & 0.172 & 0.129 & 0.226 & -9.144 & 0.000 & 2.76 \\
\hline & $0 / 5$ & & & & & & 1.26 \\
\hline Rahb & $0 / 220$ & 0.002 & 0.000 & 0.0 & -4.3 & & 1.27 \\
\hline Abera & $5 / 81$ & 0.062 & 0.026 & 0.140 & -5.894 & 0.000 & 2.49 \\
\hline Supar & $16 / 1075$ & & & & & & 2.71 \\
\hline Kerama & $14 / 6$ & 0.233 & 0.143 & 0.356 & -3.897 & & 2.66 \\
\hline Roychowe & $9 / 51$ & 0.176 & 0.094 & 0.306 & -4.194 & 0.000 & 2.60 \\
\hline Mand & & & & & & & 2.14 \\
\hline Faruqu & $711 / 12$ & 0.548 & 0.521 & 0.575 & 3.465 & 0.001 & 2.81 \\
\hline Rafi et & $37 / 12$ & 0.301 & 0.226 & 0.387 & -4.290 & 10 & 2.75 \\
\hline & & & & 0.0 & & & 2.67 \\
\hline Drom & $55 / 351$ & 0.157 & 0.122 & 0.199 & -11.462 & 0.000 & \\
\hline & & & & & & & \\
\hline & & 0.9 & $0.8 \mathrm{c}$ & 0.9 & 6.206 & 0.000 & 2.43 \\
\hline Urassa et al. & $42 / 181$ & 0.232 & 0.176 & 0.299 & -6.797 & 0.000 & 2.76 \\
\hline Garg et al. & $8 / 326$ & 0.025 & 0.012 & 0.048 & -10.287 & 0.000 & 2.61 \\
\hline & $46 / 1$ & & & & -1.708 & & \\
\hline $\mathrm{Ng} \&$ Taha & & 0.875 & 0.266 & 0.993 & 1.287 & 0.198 & 1.18 \\
\hline Glass et al. & $54 / 256$ & 0.211 & 0.165 & 0.265 & -8.612 & 0.000 & 2.77 \\
\hline & & 0.231 & 0.165 & 0.314 & -5.592 & 0.000 & \\
\hline
\end{tabular}

b

Study name

Statistics for each study

wight

.27

2.70

$\begin{array}{lllllll}\text { Sreedhara \& Mohan } & 19 / 74 & 0.257 & 0.170 & 0.368 & -3.994 & 0.000\end{array}$

$\begin{array}{llllllll}79 / 159 & 0.497 & 0.420 & 0.574 & -0.079 & 0.937 & 2.77\end{array}$

$\begin{array}{llllllll}\text { Jain et al. } & 27 / 27 & 0.982 & 0.770 & 0.999 & 2.808 & 0.005 & 1.26\end{array}$

$\begin{array}{lllllllll}\text { Torane et al. } & 55 / 439 & 0.125 & 0.097 & 0.160 & -13.479 & 0.000 & 2.78\end{array}$

upawat et al.

Dromigny et al.

Glass et al.

$\begin{array}{lllll}0.231 & 0.165 & 0.314 & -5.592 & 0.000\end{array}$

$\mid$

Event rate and $95 \% \mathrm{Cl}$

\begin{tabular}{lcccccrrr} 
& \multicolumn{2}{c}{ Total } & $\begin{array}{c}\text { Event } \\
\text { rate }\end{array}$ & $\begin{array}{c}\text { Lower } \\
\text { limit }\end{array}$ & $\begin{array}{c}\text { Upper } \\
\text { limit }\end{array}$ & Z-Value & p-Value & \multicolumn{2}{r}{ Relative } \\
weight
\end{tabular}

C

Study name

\begin{tabular}{|c|c|c|c|c|c|c|c|}
\hline & \multirow[b]{2}{*}{ Total } & & & & & & \\
\hline & & $\begin{array}{c}\text { Event } \\
\text { rate }\end{array}$ & $\begin{array}{l}\text { Lower } \\
\text { limit }\end{array}$ & $\begin{array}{c}\text { Upper } \\
\text { limit }\end{array}$ & Z-Value & $\mathrm{p}$-Value & $\begin{array}{l}\text { Relative } \\
\text { weight }\end{array}$ \\
\hline Olipher et al. & $64 / 98$ & 0.653 & 0.554 & 0.740 & 2.981 & 0.003 & 13.30 \\
\hline Dua et al. & $29 / 71$ & 0.408 & 0.301 & 0.526 & -1.534 & 0.125 & 13.21 \\
\hline Fernández-Abreu et al. & $5 / 125$ & 0.040 & 0.017 & 0.093 & -6.963 & 0.000 & 12.31 \\
\hline Irfan et al. & $5 / 233$ & 0.021 & 0.009 & 0.051 & -8.449 & 0.000 & 12.33 \\
\hline Afzali et al. & $3 / 96$ & 0.031 & 0.010 & 0.092 & -5.854 & 0.000 & 11.59 \\
\hline Supawat et al. & $3 / 22$ & 0.136 & 0.045 & 0.348 & -2.971 & 0.003 & 11.38 \\
\hline Tjaniadi et al. & $6 / 68$ & 0.088 & 0.040 & 0.183 & -5.462 & 0.000 & 12.45 \\
\hline Garg et al. & $55 / 200$ & 0.275 & 0.218 & 0.341 & -6.121 & 0.000 & 13.43 \\
\hline & & 0.135 & 0.053 & 0.302 & -3.575 & 0.000 & \\
\hline
\end{tabular}
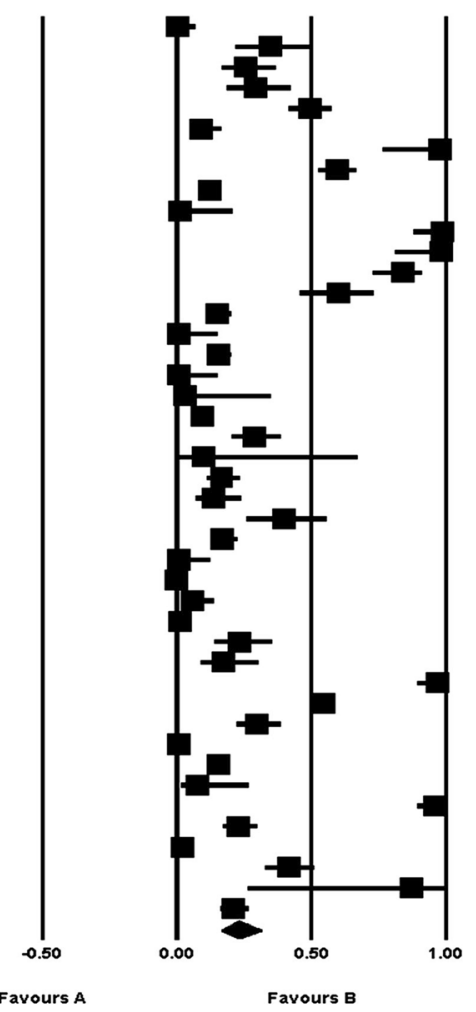

Event rate and $95 \% \mathrm{Cl}$
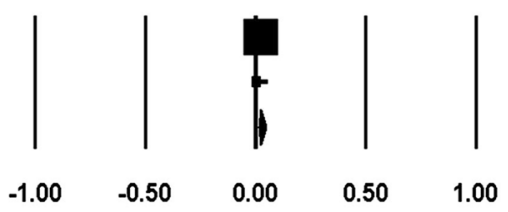

Favours A

Favours B

Event rate and $95 \% \mathrm{Cl}$
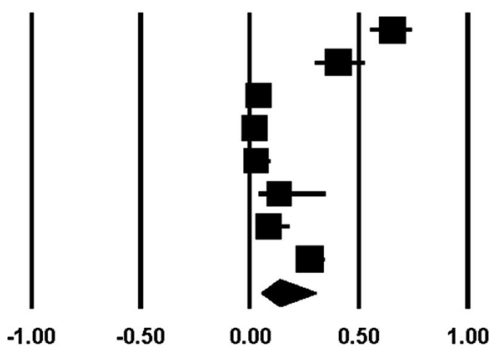

Favours A

Fig. 2 Forest plots of the meta-analysis for resistance rate of $\mathrm{V}$. cholerae serogroups $\mathrm{O} 1$ (a), $\mathrm{O} 139$ (b), and Non-O1, non-O139 (c) to tetracycline 


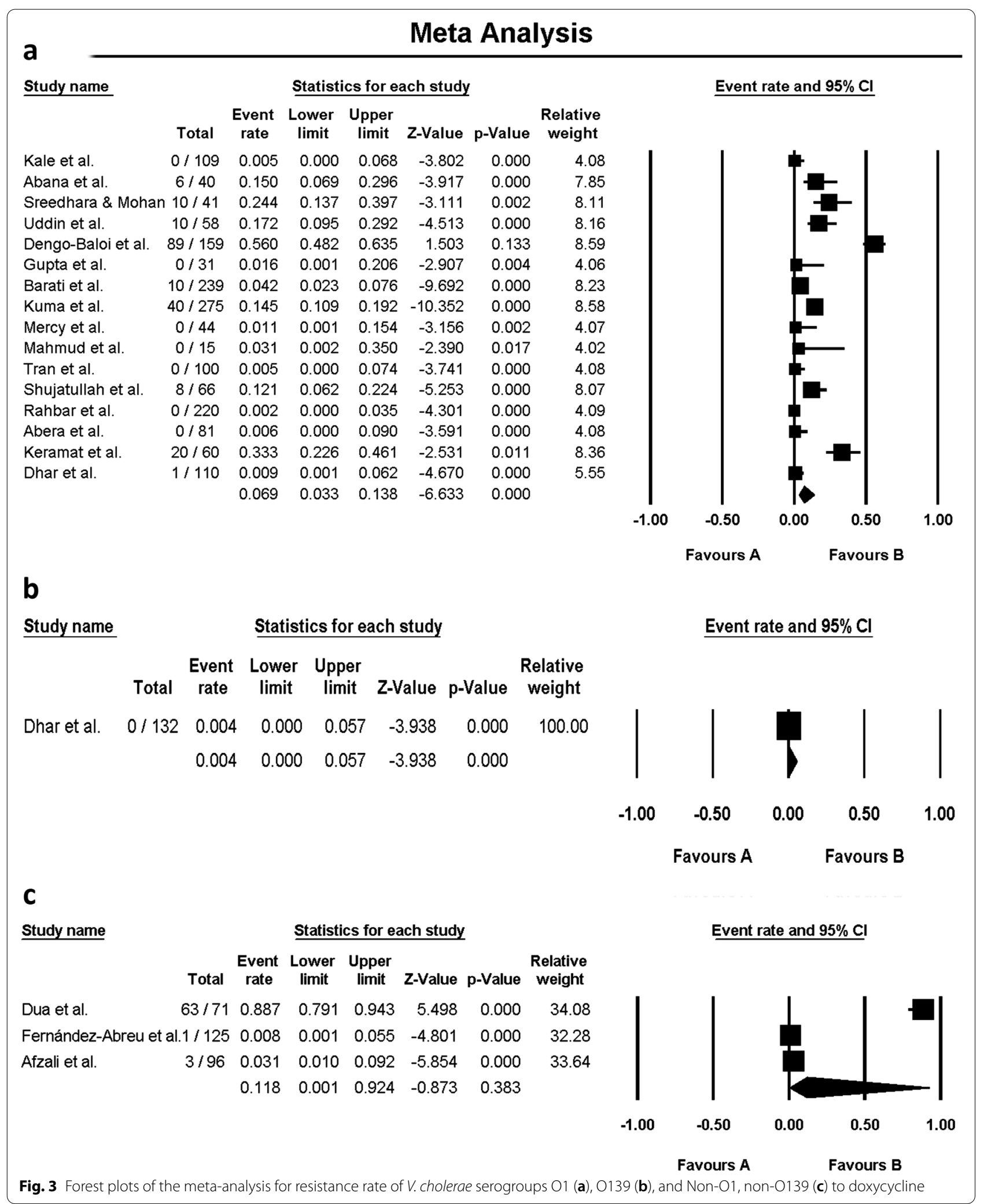


The Begg's tests did not indicate the publication bias ( $p$-value $>0.05)$. However, the Egger's tests showed some evidence of publication bias in the studies conducted on serogroup O1 (Table 2). The corresponding funnel plots of the all the analyses (except serogroup O139 in which the number of included studies was fewer than three and insufficient for application of funnel plot), are shown in Fig. 4a-d.

\section{Discussion}

The historical disease, cholera, has been endemic in south Asia, especially the Ganges delta region in Bangladesh and India, from which the disease spread outside the Indian subcontinent along trade routes causing the pandemics with high mortality rates (millions of deaths) throughout the world [2]. To date, toxigenic Vibrio cholerae (O1 serogroup) has caused seven pandemics, six of which were due to classical biotype and the seventh pandemic caused by El Tor one [37].

Although the antibiotics cannot be used as a sole treatment for the disease; however, combining fluid replacement therapy with antibiotic treatment has advantages as the antibiotics could lessen the duration of illness and reduce shedding of $V$. cholerae in the stool [4].

Tetracyclines are 'broad-spectrum antibiotics' that inhibit the bacterial 30S ribosomal subunit and consequent protein synthesis [57]. These antibiotics, particularly tetracycline and doxycycline, have long been the antibiotics of choice for treating severe cholera around the world, except for young children and pregnant women [2, 5]. However, tetracycline-resistant strains of $V$. cholerae have been emerged continuously over the years, due mainly to the extensive clinical and non-clinical uses of this class of antibiotic $[6,52]$.

By performing this systematic review and meta-analysis, it was found that the resistance rate of $V$. cholerae isolates to tetracyclines was greatly variable in various studies conducted in different geographical areas. The regional differences in resistance rate of $V$. cholerae isolates to tetracyclines may result from various exposure of patients in different populations to the antibiotics. This highlights the necessity of regional and local antibiotic susceptibility testing before antibiotic administration to avoid failure of treatment.

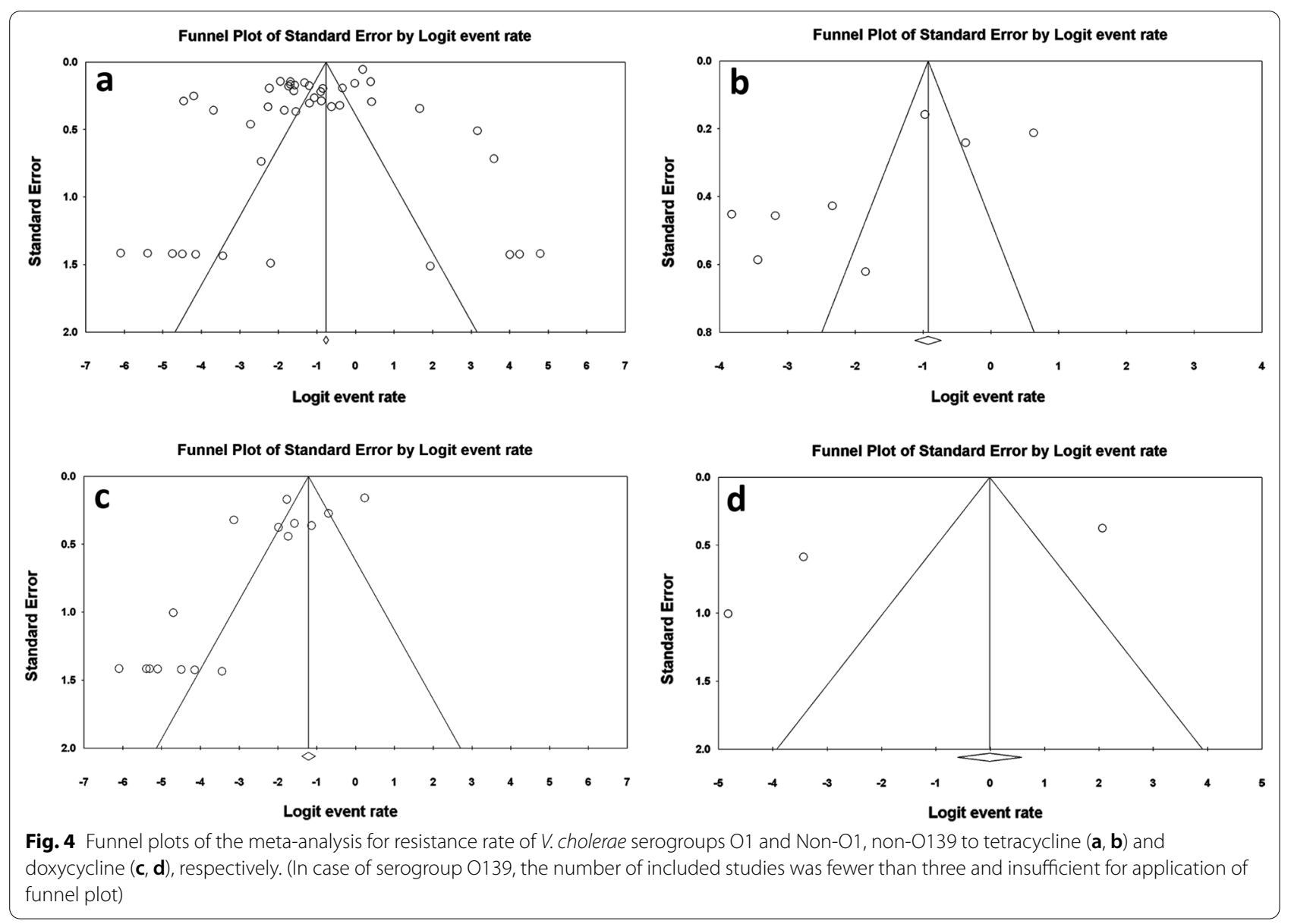


The high level of heterogeneity in the studies as well as the differences in sample sizes might impact on the analyses. To overcome this problem, the relative weight for each study was calculated and considered in the present study. Another problem in the current study was that the number of included studies conducted on the antimicrobial resistance of $\mathrm{O} 139$ and non-O1, non-O139 serogroups of $V$. cholerae was fewer than 10 and insufficient for a powerful meta-analysis and an accurate conclusion.

The results of the present meta-analysis showed that the overall resistance rate of $V$. cholerae isolates to tetracyclines (including tetracycline and doxycycline) was relatively high and between these two antibiotics, the average resistance rate to tetracycline was higher in serogroup $\mathrm{O} 1$.

Tetracycline resistance in $V$. cholerae isolates has been reported from Bangladesh since 1979 [56]. As with other antibiotics, the genes encoding resistance to tetracyclines commonly locate on mobile genetic elements such as plasmids and transposons by which the genes could be rapidly transferred and exchanged among the clinical as well as environmental strains of $V$. cholera, leading to increased resistance to these antibiotics [58]. Moreover, the antibiotic resistance determinants may be transferred and exchanged between environmental and clinical isolates of $V$. cholera through the horizontal gene transfer mechanisms [59]. These events lead to rapid increase in antibiotic resistance among the isolates.

Among the involved mechanisms of resistance to tetracyclines, the active efflux of antibiotic from bacterial cell as well as the production of ribosomal protection proteins (encoded by tet genes) are predominant in clinical settings. The other implicated mechanisms are target site mutation, decreased drug permeability, and enzymatic degradation of the antibiotic [58].

It has been evidenced that classical biotype of $V$. cholerae generally causes more severe illness compared to El Tor counterpart; in turn, the latter biotype is more adaptable and flexible in the environment, has more asymptomatic carriers, and causes higher infection to case ratio [37]. Furthermore, it has been shown that the strains of Vibrio cholerae serogroup O1 may change the biotype from Ogawa to Inaba and vice versa. Such biotype interconversion has been linked to variation in antibiotic resistance in some cases [18].

Besides tetracyclines as the first line drugs, the other antibiotic options for treatment of severe cholera include furazolidone, ciprofloxacin, erythromycin, trimethoprimsulphamethoxazole, and chloramphenicol [5]. However, the emergence of multiple antibiotic resistant strains of $V$. cholerae (displaying resistance against several antibiotics) is a major global issue and a serious problem for public health. The reasons for the appearance and development of such resistant strains may be attributed to the extensive misuse of antibiotics without proper susceptibility testing as well as the lack of an appropriate national surveillance program to monitor the bacterial resistance patterns [6]. For example, the emergence of tetracycline resistant strains causing an epidemic in Tanzania was due to the widespread use of this antibiotic for prophylaxis [50].

On the other hand, in some countries, wastewater and human excreta are routinely used for farming or in the aquaculture systems. This causes the shedding of Vibrio cholera to these environments. It is known that antibiotics are also disseminated into the environment in many ways such as excretion from humans or animals (through urine and feces), farming, and/or disposal of antimicrobials. The degradation of some antibiotics including tetracyclines takes a considerably longer time. Therefore, these antibiotics remain in water for a long period of time and gradually accumulate to reach a higher concentration. Consequently, the exposure of $V$. cholerae strains to these antibiotics in environmental settings, may lead to development and increase of resistant strains in aquatic ecosystem through natural selection. Eventually, the aquatic ecosystem as well as aquatic products serve as important reservoirs for antibiotic resistant as well as more virulent Vibrio cholerae strains capable to spread and transmit to humans via direct contact or through the food chain [59], thereby causing the epidemic infections characterized by failure in treatment.

The antimicrobial susceptibility testing according to approved CLSI guidelines (M45) is necessary prior to treatment choice [60]. However, it has been revealed that in vitro susceptibility of $V$. cholerae to antibiotics does not necessarily correlate with in vivo activity [3]. Recently, some non-antibiotic techniques have been introduced as possible alternatives to traditional antibiotics in order to control pathogens and minimize the risk of development of antibiotic-resistant strains in the environment. These possible alternatives may include inhibition of bacterial quorum sensing (quorum quenching), application of bacteriophages, and using of probiotics [59]. Moreover, it is notable that some vaccines are currently licensed or under development for prophylaxis against cholera disease in children and adults as reviewed by Shaikh et al. [61].

\section{Conclusion}

In conclusion, the results of the present study show that the overall resistance to tetracyclines, the first line treatment for cholera disease, is relatively high and prevalent among $V$. cholerae isolates, throughout the world. Hence, performing regional antimicrobial susceptibility testing according to approved CLSI guidelines prior to treatment 
choice along with monitoring and management of antibiotic resistance patterns of $V$. cholerae strains seems to be necessary. In this regard, planning the national or international surveillance programs would be helpful to reduce the emergence and propagation of antibiotic resistant strains as well as the failure of treatment.

\section{Abbreviations}

CI: Confidence interval; PRISMA: Preferred reporting items for systematic reviews and meta-analyses.

\section{Acknowledgements}

None.

\section{Authors' contributions}

MHA is the only author of the article. The author read and approved the final manuscript.

\section{Funding}

None.

\section{Availability of data and materials}

All data generated by this study have been submitted with this manuscript.

\section{Declarations}

Ethics approval and consent to participate

Not applicable.

\section{Consent for publication}

Not applicable.

\section{Competing interests}

The author declares that he has no competing interests.

Received: 24 March 2021 Accepted: 19 July 2021

Published online: 06 August 2021

\section{References}

1. Torane V, Kuyare S, Nataraj G, Mehta P, Dutta S, Sarkar B. Phenotypic and antibiogram pattern of $\mathrm{V}$. cholerae isolates from a tertiary care hospital in Mumbai during 2004-2013: a retrospective cross-sectional study. BMJ Open 2016;6:e012638

2. Sack DA, Sack RB, Nair GB, Siddique AK. Cholera. Lancet. 2004;363:223-33.

3. Kaper J, Morris G, Levine M. Cholera. Clin Microbiol Rev. 1995;8:48-86.

4. Kitaoka M, Miyata ST, Unterweger D, Pukatzki S. Antibiotic resistance mechanisms of Vibrio cholerae. J Med Microbiol. 2011;60:397-407.

5. Faruque AS, Alam K, Malek MA, Khan MG, Ahmed S, Saha D, et al. Emergence of multidrug-resistant strain of Vibrio cholerae $\mathrm{O} 1$ in Bangladesh and reversal of their susceptibility to tetracycline after two years. J Health Popul Nutr. 2007;25:241.

6. Kar SK, Pal BB, Khuntia HK, Achary KG, Khuntia CP. Emergence and spread of tetracycline resistant Vibrio cholerae O1 El Tor variant during 2010 cholera epidemic in the tribal areas of Odisha, India. Int J Infect Dis. 2015;33:45-9.

7. Moher D, Liberati A, Tetzlaff J, Altman DG. Preferred reporting items for systematic reviews and meta-analyses: the PRISMA statement. Int J Surg. 2010;8:336-41.

8. Olipher M, Johnstone M, Anne M, Joseph M, Tom M, Mwau M. Antibacterial spectrum and susceptibility of bacterial pathogens causing diarrheal illnesses: cross sectional study of patients visiting health facility in Lake Victoria Region-Kenya, East Africa. Science. 2020;2:45-58.

9. Kale SS, Bulle PA, Deshmukh DG, Tankhiwale SS, Gujar VM. An outbreak of diarrhoeal disease of El Tor Vibrio cholerae $\mathrm{O} 1$ Ogawa in and around
Yavatmal district, Maharashtra, India in 2018. Int J Community Med Public Health. 2020;7:1.

10. Abana D, Gyamfi E, Dogbe M, Opoku G, Opare D, Boateng G, et al. Investigating the virulence genes and antibiotic susceptibility patterns of Vibrio cholerae $\mathrm{O} 1$ in environmental and clinical isolates in Accra, Ghana. BMC Infect Dis. 2019;19:76.

11. Zereen F, Akter S, Sobur MA, Hossain MT, Rahman MT. Molecular detection of Vibrio cholerae from human stool collected from SK Hospital, Mymensingh, and their antibiogram. J Adv Vet Anim Res. 2019;6:451.

12. Sreedhara $H$, Mohan N. Molecular epidemiology of vibrio cholerae causing outbreaks and sporadic cholera in and around Hassan district and its antibiotic susceptibility pattern. IP Int J Med Microbiol Trop Dis. 2019:5:41-6.

13. Dua P, Karmakar A, Ghosh C. Virulence gene profiles, biofilm formation, and antimicrobial resistance of Vibrio cholerae non-O1/non-O139 bacteria isolated from West Bengal, India. Heliyon 2018:4:e01040.

14. Uddin ME, Akter T, Sultana P, Hasan MI, Lubna MA, Al Monem H, et al. Isolation, identification and antimicrobial susceptibility profile analysis of Vibrio cholerae $\mathrm{O} 1$ from stool samples of Bangladesh. Adv Microbiol. 2018:8:188-96

15. Fernández-Abreu A, Bravo-Fariñas L, Rivero-Navea G, Cabrera-Cantelar N, Nuñez-Fernández FA, Cruz-Infante Y, et al. Determinants of virulence and antimicrobial susceptibility in Non-01, Non-O139 Vibrio cholerae isolates. MEDICC Rev. 2017;19:21-5.

16. Shah R, Parveen G, Shoukat M, Khalid S, Hameed A. Isolation, identification, characterization and antibiotic susceptibility of Vibrio cholerae during 1998-99. Int J Biosci. 2017;11:135-47.

17. Dengo-Baloi LC, Semá-Baltazar CA, Manhique LV, Chitio JE, Inguane DL, Langa JP. Antibiotics resistance in El Tor Vibrio cholerae 01 isolated during cholera outbreaks in Mozambique from 2012 to 2015. PloS ONE 2017;12:e0181496.

18. Patil S, Shetty PC, Kulkarni RD, GS A, Kalabhavi A, Patil D, et al. Antibiogram and serotyping of Vibrio cholerae $\mathrm{O} 1$ isolates from a tertiary care centre in South India. J Pure Appl Microbiol 2017;11:1039-43.

19. Jain M, Kumar P, Goel A. Emergence of tetracycline resistant Vibrio cholerae $\mathrm{O} 1$ biotype El Tor serotype Ogawa with classical ctxB gene from a cholera outbreak in Odisha, Eastern India. J Pathog 2016;2016.

20. Hajia M, Saboorian R, Rahbar M. Antimicrobial resistance patterns of isolated vibrio cholerae strains. Int J Enteric Pathog. 2016;4:1-4.

21. Gupta PK, Pant ND, Bhandari R, Shrestha P. Cholera outbreak caused by drug resistant Vibrio cholerae serogroup 01 biotype ElTor serotype Ogawa in Nepal; a cross-sectional study. Antimicrob Resist Infect Control. 2016;5:23.

22. Masoumi-Asl H, Gouya MM, Rahbar M, Sabourian R. The epidemiology and antimicrobial resistance of cholera cases in Iran during 2013. Iran J Microbiol. 2016:8:232

23. Irfan S, Fasih N, Ghanchi N, Khan E. Isolation frequency and susceptibility pattern of non-O1 and non-O139 Vibrio cholerae in a tertiary health care laboratory, 1999-2012. East Mediterr Health J. 2016;22:142.

24. Afzali H, Momen-Heravi M, Razzaghi R. Antibiotic resistance of Vibrio cholerae isolates from Kashan, Iran. Int Arch Health Sci. 2016;3:67-71.

25. Ukaji D, Kemajou T, Ajugwo A, Ezeiruaku F, Eze E. Antibiotic susceptibility patterns of Vibrio cholerae $\mathrm{O} 1$ isolated during cholera outbreak in Uzebba (Edo State). Open Sci J Biosci Bioeng. 2015;2:33-6.

26. Tabatabaei SM, Salimi Khorashad A. Antimicrobial resistance patterns of Vibrio cholera strains isolated from Afghan and Iranian patients in Iran. Int J Infect 2015;2.

27. Barati H, Moradi G, Rasouli MA, Mohammadi P. Epidemiologic and drug resistance pattern of Vibrio cholerae $\mathrm{O} 1$ biotype El Tor, serotype Ogawa, in the 2011 cholera outbreak, in Alborz Province, Iran. Jundishapur J Microbiol 2015;8.

28. Mishra M, Kurhade A, Thakar Y, Kurhade G, Vaillant AJ, Lakhdive S, et al. Occurrence of a cholera outbreak in central India. Am J Infect Dis Microbiol. 2015;3:141-3.

29. Kuma GK, Opintan JA, Sackey S, Nyarko KM, Opare D, Aryee E, et al. Antibiotic resistance patterns amongst clinical Vibrio cholerae $\mathrm{O} 1$ isolates from Accra, Ghana. Int J Infect Control 2014;10.

30. Mercy N, Mohamed AA, Ng'ang'a Zipporah GC, Pazhani GP, Ramamurthy $\mathrm{T}$, Boga $\mathrm{HI}$, et al. Phenotypic and genetic characterization of Vibrio cholerae $\mathrm{O} 1$ isolated from various regions of Kenya between 2007 and 2010. Pan Afr Med J 2014;19. 
31. Mahmud ZH, Islam S, Zaman RU, Akter M, Talukder KA, Bardhan PK, et al. Phenotypic and genotypic characteristics of Vibrio cholerae $\mathrm{O} 1$ isolated from the Sierra Leone cholera outbreak in 2012. Trans R Soc Trop Med Hyg. 2014;108:715-20.

32. Murhekar M, Dutta S, Ropa B, Dagina R, Posanai E, Rosewell A. Vibrio cholerae antimicrobial drug resistance, Papua New Guinea, 2009-2011. Western Pac Surveill Response J. 2013;4:60

33. Tran HD, Alam M, Trung NV, Van Kinh N, Nguyen HH, Pham VC, et al. Multi-drug resistant Vibrio cholerae $\mathrm{O} 1$ variant El Tor isolated in northern Vietnam between 2007 and 2010. J Med Microbiol. 2012;61:431.

34. Sang WK, Oundo V, Schnabel D. Prevalence and antibiotic resistance of bacterial pathogens isolated from childhood diarrhoea in four provinces of Kenya. J Infect Dev Ctries. 2012;6:572-8.

35. Mandal J, Dinoop K, Parija SC. Increasing antimicrobial resistance of Vibrio cholerae OI biotype EI Tor strains isolated in a tertiary-care centre in India. J Health Popul Nutr. 2012;30:12.

36. Shujatullah F, Shukla I, Gupta R, Khan PA, Khan F. Emergence of antimicrobial resistance in Vibrio cholerae of North India. Int Res J Microbiol. 2012;3:136-9.

37. Borkakoty B, Biswas D, Devi U, Yadav K, Mahanta J. Emergence of classical ctxB genotype 1 and tetracycline resistant strains of Vibrio cholerae $\mathrm{O} 1 \mathrm{El}$ Tor in Assam, India. Trans R Soc Trop Med Hyg. 2012;106:382-6.

38. Das S, Choudhry S, Saha R, Ramachandran VG, Kaur K, Sarkar B. Emergence of multiple drug resistance Vibrio cholerae 01 in East Delhi. J Infect Dev Ctries. 2011;5:294-8.

39. Karki R, Bhatta D, Malla S, Dumre S, Upadhyay B, Dahal S, et al. Resistotypes of Vibrio cholerae 01 Ogawa Biotype El Tor in Kathmandu, Nepal. Nepal Med Coll J. 2011;13:84-7.

40. Rahbar M, Zahraei M, Omidvarnia A, Afshani MT, Glami M, Sabourian R, et al. Survey of epidemiology and bacteriology features of cholera in Iran. Asian Pac J Trop Med. 2010:3:45-7.

41. Abera B, Bezabih B, Dessie A. Antimicrobial suceptibility of V. cholerae in north west, Ethiopia. Ethiop Med J 2010:48:23-8.

42. Supawat K, Huttayananont S, Sawanpanyalert P, Aswapokee N, Mootsikapun P. Antimicrobial resistance surveillance of Vibrio cholerae in Thailand from 2000 to 2004. J Med Assoc Thai. 2009;92:S82-6.

43. Keramat F, Hashemi S, Mamani M, Ranjbar M, Erfani H. Survey of antibiogram tests in cholera patients in the 2005 epidemic in Hamadan, Islamic Republic of Iran. East Mediterr Health J. 2008;14:768-75.

44. Roychowdhury A, Pan A, Dutta D, Mukhopadhyay AK, Ramamurthy T, Nandy RK, et al. Emergence of tetracycline-resistant Vibrio cholerae $\mathrm{O} 1$ serotype Inaba, in Kolkata, Indai. Jpn J Infect Dis. 2008:61:128.

45. Mandomando I, Espasa M, Vallès X, Sacarlal J, Sigaúque B, Ruiz J, et al. Antimicrobial resistance of Vibrio cholerae $O 1$ serotype Ogawa isolated in Manhica District Hospital, southern Mozambique. J Antimicrob Chemother. 2007;60:662-4.

46. Rafi S, Rafi S, Qureshi A, Saeed W, Ali A, Ahmadani M, et al. Changing epidemiology and sensitivity pattern of vibrio cholerae at Rawalpindi. Pak J Med Sci. 2004;20:357-60.
47. Tjaniadi P, Lesmana M, Subekti D, Machpud N, Komalarini S, Santoso W, et al. Antimicrobial resistance of bacterial pathogens associated with diarrheal patients in Indonesia. Am J Trop Med Hyg. 2003;68:666-70.

48. Dromigny J-A, Rakoto-Alson O, Rajaonatahina D, Migliani R, Ranjalahy J, Mauclère $P$. Emergence and rapid spread of tetracycline-resistant Vibrio cholerae strains, Madagascar. Emerg Infect Dis. 2002;8:336.

49. Sabeena F, Thirivikramji G, Radhakutty G, Indu P, Singh D. In vitro susceptibility of Vibrio cholerae $\mathrm{O} 1$ biotype El Tor strains associated with an outbreak of cholera in Kerala, Southern India. J Antimicrob Chemother. 2001;47:361-2.

50. Iwanaga M, Insisiengmay S, Higa N, Sisavath L. Tetracycline resistant and polymyxin B sensitive Vibrio cholerae $\mathrm{O} 1 \mathrm{El}$ Tor isolated from the recent epidemics. Jpn J Trop Med Hyg. 2000;28:15-8.

51. Urassa W, Mhando Y, Mhalu F, Mgonja S. Antimicrobial susceptibility pattern of Vibrio cholerae 01 strains during two cholera outbreaks in Dar Es Salaam, Tanzania. East Afr Med J 2000;77.

52. Garg P, Chakraborty S, Basu I, Datta S, Rajendran K, Bhattacharya T, et al. Expanding multiple antibiotic resistance among clinical strains of Vibrio cholerae isolated from 1992-7 in Calcutta, India. Epidemiol Infect. 2000;124:393-9.

53. Ranjit K, Nurahan M. Tetracycline resistant cholera in Kelantan. Med J Malaysia. 2000;55:143-5.

54. Dhar U, Bennish ML, Khan WA, Seas C, Khan EH, Albert MJ, et al. Clinical features, antimicrobial susceptibility and toxin production in Vibrio cholerae 0139 infection: comparison with V. cholerae $\mathrm{O} 1$ infection. Trans R Soc Trop Med Hyg 1996;90:402-5.

55. Ng P, Taha M. Tetracycline resistant Vibrio cholerae in pilgrims returning from Mecca. Med J Malaysia 1994;49:195.

56. Glass RI, Huq I, Alim A, Yunus M. Emergence of multiply antibiotic-resistant Vibrio cholerae in Bangladesh. J Infect Dis. 1980;142:939-42.

57. Zhanel GG, Esquivel J, Zelenitsky S, Lawrence CK, Adam HJ, Golden A, et al. Omadacycline: a novel oral and intravenous aminomethylcycline antibiotic agent. Drugs 2020;1-29.

58. Thaker M, Spanogiannopoulos P, Wright GD. The tetracycline resistome. Cell Mol Life Sci. 2010;67:419-31.

59. Loo KY, Letchumanan V, Law JWF, Pusparajah P, Goh BH, Ab Mutalib NS, et al. Incidence of antibiotic resistance in Vibrio spp. Rev Aquac. 2020:12:2590-608.

60. CLSI. Methods for antimicrobial dilution and disk susceptibility testing of infrequently isolated or fastidious bacteria. 3rd ed. CLSI guideline M45. CLSI Wayne, PA: Clinical and Laboratory Standards Institute; 2016.

61. Shaikh H, Lynch J, Kim J, Excler J-L. Current and future cholera vaccines. Vaccine. 2020;38:A118-26.

\section{Publisher's Note}

Springer Nature remains neutral with regard to jurisdictional claims in published maps and institutional affiliations.

\footnotetext{
Ready to submit your research? Choose BMC and benefit from:

- fast, convenient online submission

- thorough peer review by experienced researchers in your field

- rapid publication on acceptance

- support for research data, including large and complex data types

- gold Open Access which fosters wider collaboration and increased citations

- maximum visibility for your research: over $100 \mathrm{M}$ website views per year
}

At BMC, research is always in progress.

Learn more biomedcentral.com/submissions 